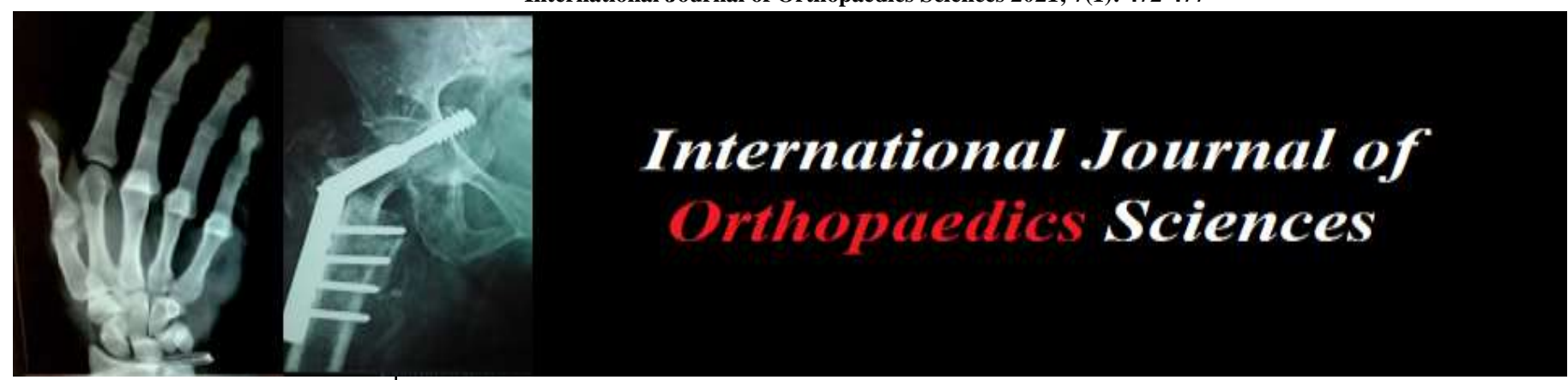

E-ISSN: 2395-1958

P-ISSN: 2706-6630

IJOS 2021; 7(1): 472-477

(C) 2021 IJOS

www.orthopaper.com

Received: 09-11-2020

Accepted: 16-12-2020

Abhishek Kumar

ESIC PGIMSR and Model

Hospital Delhi, Delhi, India

Akhilesh Kumar

Muzaffarnagar Medical College (MMC), Uttar Pradesh, India

Corresponding Author:

Abhishek Kumar

ESIC PGIMSR and Model

Hospital Delhi, Delhi, India

\section{Outcome analysis of fluoroscopically guided selective spinal nerve root block for lumbar radicular pain in tertiary care centre}

\section{Abhishek Kumar and Akhilesh Kumar}

DOI: https://doi.org/10.22271/ortho.2021.v7.i1h.2528

Abstract

Introduction: Lumbar radiculopathy is a common complaint of patients presenting to spine clinic. A variety of conditions can lead to compression of the nerve roots, the most common cause is disc herniation. Selective Nerve Root block (SNRB) injections have been used in everyday clinical practice.

Methods: Fifty patients with lumbar radicular pain were enrolled in the study as per the inclusion criteria. Patients were assessed clinically, neurologic evaluation done and pain quantified by Visual analogue scale (VAS) and the Oswestry Disability Index (ODI). They were reassessed after SNRB immediately, at 3 weeks and 6 weeks using the same outcome measures.

Results: Majority of patients (40 out of 49) demonstrated improvement after SNRB).The degree of improvement in the VAS was significantly greater and statistically significant $(P<0.001)$. The degree of improvement in the ODI was also statistically significant $(P<0.001)$ after 3 and 6 weeks.

Conclusion: Spinal nerve root block is effective in the selected group of patients of lumbar radicular pain and should be recommended as an alternative treatment to surgery for this condition along with physiotherapy and medications.

Keywords: Disc herniation, lumbar radiculopathy, Selective Nerve Root block

\section{Introduction}

Lumbar radiculopathy is a common complaint of patients presenting to spine clinic. Lumbar radiculopathy refers to symptoms of pain, tingling, numbness or weakness that travel down the low back and into the leg or extremity ${ }^{[1,2]}$. Lumbar radiculopathy may occur as a result of disc herniation, degenerative disc disease, arthritis of the lumbar spine, trauma and injury to the lumbar spine, but the most common cause is disc herniation. It is often diagnosed by history and symptoms, which include pain, burning, tingling or numbness that radiate from the lower back and buttock around the back of the thigh to cause low back pain, buttock pain or most commonly, hip pain ${ }^{[1,2]}$.

Since its first description by Weber et al. ${ }^{[1]}$ in 1934 , lumbar disc herniation is one of the few abnormalities in the lumbar spine, where a clear relationship between the morphological alteration and pain seems to exist. While pure mechanical compression was considered previously as a source of sciatica, there is increasing evidence that chemical irritation of the nerve root plays an essential and perhaps even more important role ${ }^{[3,4]}$. Autoimmune responses, microvascular changes and inflammatory reactions are discussed as potential causes of this phenomenon ${ }^{[5-8]}$.

Nucleus pulposus tissue has inflammatory properties, which lead to an intraneural oedema, a very important factor in the pathogenesis of sciatic pain ${ }^{[6]}$. The negative effect of nucleus pulposus on the nerve root can be significantly reduced by the application of methylprednisolone ${ }^{[9]}$. The compromising of the nerve conduction velocity by nucleus pulposus tissue seems to be self-limiting ${ }^{[9]}$.

Brown et al. ${ }^{[9]}$ has shown in an animal model, that this effect is most pronounced after seven days and spontaneously normalises within a two month period. These experimental findings may explain, why sciatica has a favourable natural history ${ }^{[11]}$. Surgery in patients presenting with a radiculopathy with or without minor neurological sensory/motor deficit is only required, if the initial pain cannot be well controlled by non-operative means. 
Otherwise, surgery is not required because spontaneous recovery can be expected ${ }^{[10-12]}$.

The recent researches that have been conducted within the last decade show that a definite trend towards non-surgical management of lumbosacral disc herniations with radicular symptoms has occurred ${ }^{[6]}$. Non-surgical treatment of lumbar radicular pain includes non-steroid anti-inflammatory drugs (NSAIDs), analgesics, oral or parenteral steroids, therapeutic exercises, the epidural injections and selective epidural nerve root blocks. The treatment options are considerable and yet the outcomes associated with many treatments are either questionable or not well investigated ${ }^{[4]}$. One of the postulated causes for radicular leg pain is inflammation of the nerve root. The role of steroids in such a condition is to inhibit the inflammatory reaction and subsequently reduce pain ${ }^{[4]}$.

Although sciatica is usually caused by a mechanical abnormality, pathophysiological considerations provide a sound rationale for local corticosteroid treatment: sciatica due to disc herniation resolves within one month in $70 \%$ of nonsurgically-treated patients ${ }^{[1]}$, while after one year as many as $90 \%$ of patients are improved ${ }^{[3]}$; several lines of evidence suggest that biochemical factors may contribute to sciatica ${ }^{[6]}$. Magnetic resonance studies have demonstrated postgadolinium enhancement consistent with an inflammatory response ${ }^{[8]}$. There is also evidence that this treatment should be started early to prevent persistent nerve root pain due to peripheral and central sensitization ${ }^{[9]}$.

There are some complications of fluoroscopically guided spinal injection procedures (including EBI, SNRB, facet joint blocks,), like subarachnoid needle placement, allergic reactions to contrast material, allergic reaction to local anesthetics, vasovagal response with severe bradycardia, and pain exacerbation ${ }^{[13]}$.

It's a very effective method with high success rate and the economic burden to the patients in terms of the cost of the drugs used can also be decreased. Also noteworthy is the fact that the patient can be immediately discharged, hence shortening the hospital stay of the patient.

\section{Null Hypothesis}

There is no improvement in clinical outcome of fluoroscopic guided selective nerve root block for lumbar radicular pain.

\section{Objectives of study}

\section{General objective}

Outcome analysis of fluoroscopic guided selective nerve root block for lumbar radicular pain.

\section{Specific objective}

1. To assess the demographic profile of patients with lumbar radiculopathy presenting to our centre (TUTH hospital).

2. To assess the disc herniation morphology.

3. To correlate disc morphology and effect of SNRB.

\section{Methods and methodology}

Type of study

A prospective, observational study.

\section{Place of study}

Tribhuvan university teaching hospital, Kathmandu, Nepal: Operation Theatre (OT) and Out Patient Department (OPD) of Orthopedics and Trauma surgery, Spine unit.

\section{Duration of study}

24 months (March 2015 to February 2017).

\section{Inclusion criteria}

1. Age group 20-80

2. Clear identification of an affected nerve root with MRI proven herniated intervertebral disc L4-L5 or L5-S1 level with evidence of nerve root compromise with or without spinal canal stenosis with foraminal narrowing.

3. Absence of progressive neurological deficit.(MRC Grade of 3 or more)

4. VAS score of 7 or more.

5. Oswestery disability score of $60-100 \%$.

6. Patients having radiculopathy with VAS $<7$ and ODS $<60 \%$ not responding to pain medication and physiotherapy for 2 weeks.

\section{Exclusion criteria}

1. Patients having motor deficit that is MRC grade less than 3 or cauda equina syndrome.

2. Patients with history of previous spinal surgery.

3. Patients having multiple level herniated discs except L4L5 and L5-S1.

4. Patients having other spinal pathologies like spondylolisthesis, kyphosis, scoliosis, fractures etc.

5. Patients with previous history of allergy to steroids.

6. Patients with bleeding disorders.

\section{Sample size}

The number of patients of lumbar radiculopathy presenting to the OPD department of TUTH and enrolled in my study of 2 year was 50 which is calculated as follows.

Sample size $(\mathrm{n})=\mathrm{Zq}(1-\mathrm{q}) / \mathrm{p} 2$

Prevalence in population $(\mathrm{q})=5 \%=0.05$

Precision $(\mathrm{p})=5 \%=0.05$

So $\mathrm{n}=1.96 * 0.05 * 0.95 / 0.05 * 0.05=40$

Drop out rate $=10 \%=4$ so sample size $=40+4=44$ (50 taken)

\section{Statistical analysis}

Data was analyzed using Microsoft excel 2007 and SPSS 20.0 for Windows. Results were expressed as percentages, mean \pm standard deviation for variables. The level of significance was set at $5 \% \quad(\mathrm{P} \leq 0.05)$ and, for high significance $0.1 \%$ ( $\mathrm{P}$ $\leq 0.001)$. Various tables, charts, bar diagrams were used using independent $t$ tests.

\section{Methods}

\section{Pre treatment evaluation of patients}

Patients were selected as described in selection criteria. Consent was taken from all eligible candidates after fully explaining nature of study. A detailed proforma was filled up with identification of the patients including address, telephone number (if possible) separately for every participant. Proper history of pain with its duration, onset, location, radiation, effect of position, aggravating and relieving factors and any risk factors were asked. History of bowel and bladder, any sensory and motor loss were asked. Past history of any disease (e.g. tuberculosis, bleeding disorders), medication and the effect of any previous treatment was assessed. Patients job description along with daily activities and work leisure activities hampered and their anxiety towards back pain with sciatica was noted. Patients were not admitted but explained and prepared to spend few hours to recover from the procedure.

Patients were seen and assessed before the procedure by clinical examination and using the Visual Analogue Score (VAS) and ODI and standard detailed neurological evaluation was done under supervision of study guide and co guide. 
Radicular leg pain, and not low back pain, was assessed.

After the systemic examination, the hip and sacro-iliac joint were thoroughly examined as any pathology in these areas may be confused with pain of spinal origin.

X-rays and MRI scans of spine were obtained before the root blocks as per department protocol.

Steps of the procedure are ${ }^{[16]}$ :

1. The nerve root block was performed under sterile conditions with an image intensifier. The technique described by Bogduk et al was used.

2. The target point was a "safe triangle" i.e., above the exiting nerve root and below the corresponding pedicle.
The skin was anaesthetised with $2-3 \mathrm{ml}$ lignocaine $2 \%$.

3. A spinal needle $(22 \mathrm{G})$ was inserted paramedian through the skin and muscles in a cranio-medial direction until a bony contact was encountered. This method allowed advancing the needle in a safe triangle without contact to the nerve root.

4. After verification of a correct needle positioning under biplanar image intensifier control, $1 \mathrm{ml}$ of iodinated dye was injected and traversing root was visualised and then $1 \mathrm{ml}$ of methylprednisolone (40 mg) plus $1 \mathrm{ml}$ of $2 \%$ lignocaine was injected. With this technique the nerve roots L5-S1 could be targeted.

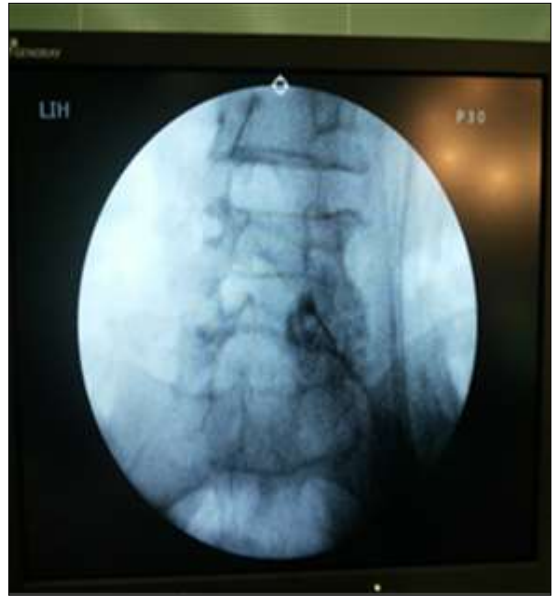

Fig 1: Dye given and root visualized

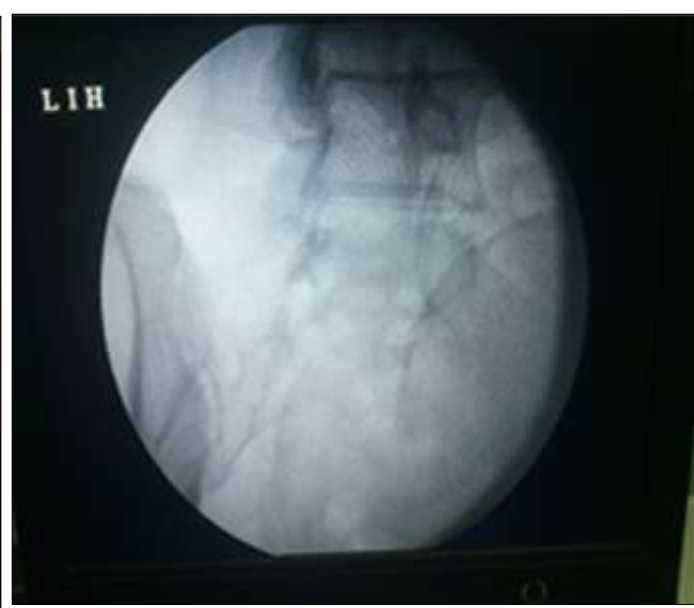

Fig 2: Verification of level using C-Arm

Immediate post injection evaluation included VAS. During the first, and second follow up patients were assessed for Visual Analogue Score ${ }^{[24]}$, Oswestry Disability Index score.

\section{Results}

Total of 50 patients were included in our study and were analyzed to assess the demographic profile of patients with lumbar radiculopathy. In 9 patients, the nerve root block did not show a sufficient pain reduction despite a correct periradiculogram. 1 out of 50 patients was lost to subsequent follow up.

Table 1: Age, gender characteristics and disc morphology

\begin{tabular}{|c|}
\hline Age in years (Mean \pm S. D) $41.92 \pm 15.10($ Min age $=22$, Max age $=76)$ \\
\hline Sex $(\mathrm{N}=50) \mathrm{M}=31, \mathrm{~F}=19$ \\
\hline Mean duration of symptoms=4 months \\
\hline Disc level (N=50) L4 -L5 $=25(50 \%), \mathrm{L} 5-\mathrm{S} 1=17(34 \%)$, Both=8(16\%) \\
\hline Stage of disc herniation $(\mathrm{N}=50)$ bulge $=8(16 \%)$ protrusion=36(72\%) extrusion=6(12\%) \\
\hline Type of disc herniation $(\mathrm{N}=50)$ central=8(16\%) paramedian=18(36\%) both=24(48\%) \\
\hline
\end{tabular}




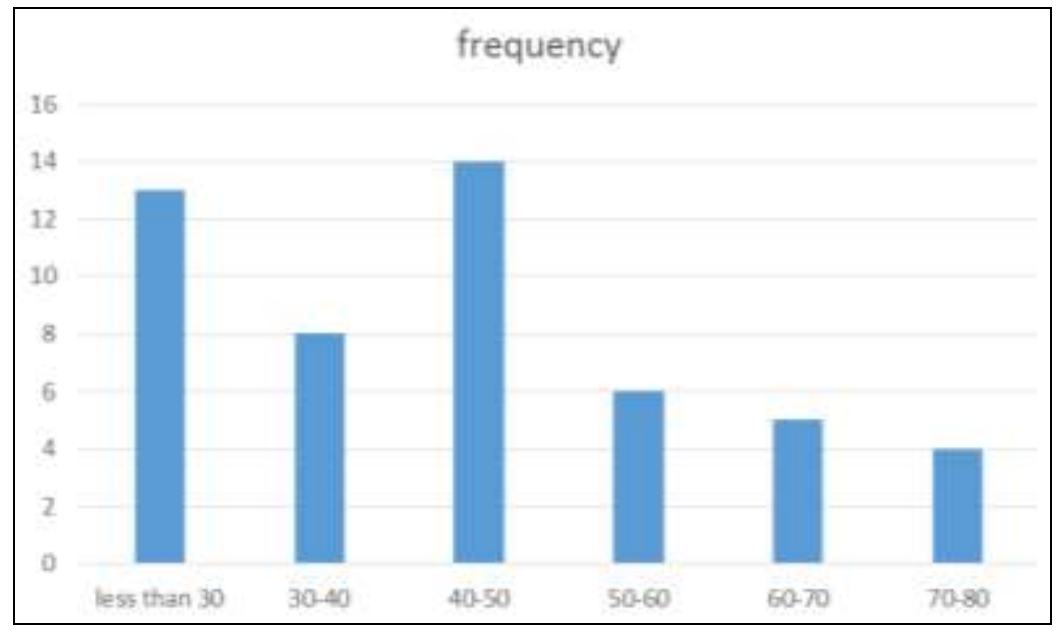

Table 2: Age distribution

$13(26 \%)$ patients belonged to less than 30 years age group, $8(16 \%)$ belonged to $30-40$ years age group, $14(28 \%)$ were in $40-50$ age group, $6(12 \%)$ were in the 50-60 age group,
$5(10 \%)$ in the $60-70$ age group and $4(8 \%)$ in the $70-80$ age group. Comparison between the mean VAS at presentation, immediately after SNRB, 3 weeks and 6 weeks follow up:

Table 3: Overall comparison of vas score

\begin{tabular}{|c|c|c|c|c|}
\hline & No of patients & Mean & Std. Deviation & \\
\hline Pre VAS & 50 & 7.98 & 1.45 & \\
\hline VAS immediately & 50 & 4.32 & 2.45 & $(p<0.001)$ \\
\hline VAS after 3 weeks & 40 & 3.00 & 1.632 & $(p<0.001)$ \\
\hline VAS after 6 weeks & 40 & 2.42 & 1.21 & $(p<0.001)$ \\
\hline
\end{tabular}

The mean VAS was 7.98 at presentation with a S.D of 1.45 , immediately after SNRB was 4.32 with a S.D of 2.45 , after 3 weeks was 3.00 with a S.D of 1.632 and after 6 weeks was 2.42 with a S.D of 1.21 which was statistically significant $(p<$
$0.001)$

Comparison between the mean ODI at presentation, after 3 weeks and after 6 weeks after SNRB

Table 4: Overall comparison between ODI score

\begin{tabular}{|c|c|c|c|c|}
\hline & No of patients & Mean & Std. Deviation & \\
\hline Pre ODI & 50 & $68.14 \%$ & 20.00 & \\
\hline ODI after 3 weeks & 40 & $18.73 \%$ & 11.89 & $(p<0.001)$ \\
\hline At 6 week ODI & 40 & $10.24 \%$ & 6.05 & $(p<0.001)$ \\
\hline
\end{tabular}

The mean ODI at presentation was found to be $68.14 \%$ with a S.D of 20.00 , after 3 weeks was $18.73 \%$ with a SD of $11.89 \%$ and after 6 weeks was $10.24 \%$ with a SD of 6.05 with a better outcome which was statistically significant ( $p$ $<0.001)$.

In our study at 6 weeks' follow-up, 40 of 49(81.63\%) patients reported successful pain reduction. 1 patient was lost to subsequent follow ups. Root blocks were failed in $9(18.36 \%)$ patients because in these patients because there was no relief of pain as well as no improvement in VAS immediately after block. Mean VAS score immediately in successful root block patients (40) was 7.88 with a S.D of 1.453 . Mean VAS score immediately in failed root block patients (9 out of 49) was 8.44 with a S.D of 1.590. Mean ODI score in successful root block patients (40 out of 49) was 18.73 with a S.D of 11.89 at 3 weeks follow up

\section{Discussion}

Lumbar radiculopathy is a common complaint of patients in Nepal presenting to spine clinic. Symptoms of pain, tingling, numbness or weakness radiating down the low back and into the extremity are associated with lumbar radiculopathy. Common causes are degenerative disc disease, arthritis of the lumbar spine, and through trauma or injury to the lumbar spine, but the most common cause is disc herniation ${ }^{[1,2]}$. There are surgical and nonsurgical treatments of lumbar radicular pain. Non -surgical treatments includes non steroid anti inflammatory drugs (NSAIDS), analgesics, oral or parenteral steroids, therapeutic exercises and epidural injections and selective nerve root blocks ${ }^{[6]}$. Selective nerve root blocks are an effective and less invasive intervention, and serve as an adjunct to non-operative treatment ${ }^{[16]}$.

In the gender distribution, in my study males (62\%) as compared to females $(38 \%)$ are more predominant in our study. In similar to our gender distribution, in a study by Christian W. A. Pfirrmann, and Patrick A et al. ${ }^{[16]}$ out of 36 patients, 13 were women and 23(64\%) were men and thus predominantly were males. This predominance in males can be explained by that males are more involved in the outdoor and heavy weight activities.

In our study group the minimum age of the patient was 22 years and a maximum of 76 years with a mean age of 41.92 years and SD of 15.10. In age related complaints maximum patient $28 \%$ fall in $41-50$ years followed by $26 \%$ and $16 \%$ in 21-30 and 31-40 years respectively. Whereas age group of 61 - 70 years has only $10 \%$ of the total patients.) In a study by 
Christian W. A. Pfirrmann, and Patrick A et al. ${ }^{[17]}$ in Swiss population in 2001, mean age was 52 years and age range was 22-88. In a study by Arvind Pandey et al. ${ }^{[22]}$ in Indian population in 2016, the maximum patients were in the age group of 30 to 40 years and the average age of patient in was 35.37 (18-72) years. This age is most common for disc degeneration predisposing to disc herniation leading to lumbar radiculopathy.

In this study we found the commonest level of affected intervertebral disc prolapsed was L4-L5(50\%) followed by L5-S1(34\%). Biomechanically $80-90 \%$ of the movements of the lumbar spine occur at L4-L5 and L5-S1 intervertebral discs as well as disc degeneration causing lumbar radicular pain are common at these levels hence these levels found to be prominent in my study.

In our study at 6 weeks' follow-up, 40 of $49(81.63 \%)$ patients reported successful pain reduction. In the study by Martin Naroznya et al. Marco Zanetti et al. and Norbert Boos et al. ${ }^{[17]}$ in Swiss population in 2001, at 2 to 3 weeks' followup, 26 of 30 patients reported successful pain reduction. There were no complications, in particular no infections, nerve root injuries or bleeding events.

In a prospective study in Scotland in 1997, Weiner and Fraser et al. ${ }^{[14]}$ investigated the success of nerve root blocks in 30 patients with foraminal and extraforaminal disc herniation. They found an immediate pain reduction in 27 patients, of whom only 3 required surgery because of recurrent leg pain, whilst 2 individuals were lost to follow-up. In total in this study, 22 of 28 patients $(79 \%)$ had a substantial and permanent pain reduction during a 1-10 year follow up.

In a study by Rahul Madhukar Salunkhe et al. in Indian population in $2016^{\text {[23] }}$, out of fifty patients of radicular pain selected after thorough clinical and radiological examination and subjected to SNRB, 45 (90\%) had enjoyed complete pain relief after SNRB immediately and $31(62 \%)$ of which were completely symptom-free at the end of 1 year. 41 patients $(82 \%)$ had excellent result, $4(8 \%)$ patients had good result, 2 (4\%) patients had fair, and $3(6 \%)$ had poor results. Cuckler et al. ${ }^{[18]}$ treated 73 patients with radicular leg pain either with methylprednisolone acetate and did not observe an effect of the steroids after an average of 20 months between both groups. The key issue of the local steroid injection is a shortterm relief and not a long-term effect because of the benign natural history of this disease ${ }^{[15]}$.

In a study by Christian W. A et al. and Pfirrmann et al. ${ }^{[17]}$ in Swiss population in 2001, seventy-five percent of patients in the study experienced pain relief 15 minutes after the procedure, and $86 \%$ reported a benefit after 2 weeks. Eightysix percent of patients in the study had at least some pain relief after 2 weeks, which compares favorably to the results in a study in which ESI was used, which demonstrated that $62 \%$ of patients felt better 2 weeks after the procedure ${ }^{[19]}$.

In our study, there was no statistically significant difference in VAS and ODI score for age less than 35 and more than equal to 35 years, different stages of disc herniation different levels of disc herniation, different types of disc herniation, duration of symptoms, laterality of radicular pain and effect of SNRB.

In our study 1 patient was lost to subsequent follow ups. Root blocks were failed in $9(18.36 \%)$ patients. The reason of the failure of the blocks are 7 of the 9 patients had large herniated discs. 4 of the seven failed block patients had central type of disc herniation and 5 had both central and paramedian type of disc herniation. 5 of the seven patients with failed blocks had symptoms of duration more than 4 months. Out of the 9 patients with failed blocks, 6 had protruded discs and 3 had extruded type of discs. Other reasons can be faulty technique, wrong level, and congenital root anomalies, old age etc. So the SNRBs have higher success rates with paramedian type of disc herniations and bulged type and in those who are of young age and have duration of symptoms less than 4 months. In a prospective series of 139 diagnostic SNRB procedures by Bogduk et al. No major complications were recorded ${ }^{[19]}$. In a series of 888 fluoroscopically guided spinal injection procedures (including EBI, SNRB, facet joint blocks), eight reversible complications occurred: three cases of subarachnoid needle placement, two allergic reactions to contrast material, one allergic reaction to local anesthetics, one vasovagal response with severe bradycardia, and one case of pain exacerbation ${ }^{[13]}$. There is a potential risk of infection with spinal injections. In my study, no case of infection with SNRB was reported and no complications occurred. In general, spinal injections are safe and accurate when performed with imaging guidance ${ }^{[20]}$. Contraindications to the procedure are bleeding diathesis, suspected local infection (which could be unmasked by the steroids), and adrenal function that may be suppressed for 2-3 weeks ${ }^{[21]}$. In my study, major reversible complications or persistent structural damage to the nerve root has not been reported with SNRBs.

\section{Conclusion}

Spinal nerve root block is effective in the selected group of patients of lumbar radicular pain atleast for short term and should be recommended as an alternative treatment to surgery for this condition along with physiotherapy and medications

\section{References}

1. Weber H. Spine Update The Natural History of Disc Herniation and the Influence of Intervention. Spine. 1994;19(19):2234-2238.

2. Degenerative Disc Disease and Sciatica Treatment, Symptoms \& Causes [Internet]. Medicine Net. 2020. [Cited 28 July 2020]. Available from: https://www.medicinenet.com/degenerative_disc/article.h tm

3. Saal J, Saal J. Nonoperative Treatment of Herniated Lumbar Intervertebral Disc with Radiculopathy. Spine 1989;14(4):431-437.

4. Feuerstein M, Marcus S, Huang G. National trends in nonoperative care for nonspecific back pain. The Spine Journal 2004;4(1):56-63.

5. Galloway H. Image-guided spinal injection for diagnosis and therapy. Australasian Radiology 2003;47(3):219-225.

6. Weinstein S, Herring S. Lumbar epidural steroid injections. The Spine Journal 2003;3(3):37-44.

7. Goupille P, Jayson M, Valat J, Freemont A. The role of inflammation in disk herniation-associated radiculopathy. Seminars in Arthritis and Rheumatism 1998;28(1):60-71.

8. Komori H, Okawa A, Haro H, Muneta T, Yamamoto H, Shinomiya K. Contrast-Enhanced Magnetic Resonance Imaging in Conservative Management of Lumbar Disc Herniation. Spine 1998;23(1):67-73.

9. Rydevik B, Brown M, Lundborg G. Pathoanatomy and Pathophysiology of Nerve Root Compression. Spine 1984;9(1):7-15.

10. Beliveau P. A Comparison between Epidural Anaesthesia with and Without Corticosteroid in the Treatment of Sciatica. Rheumatology 1971;11(1):40-43.

11. Burn M, Langdon L. Lumbar Epidural Injection for the Treatment of Chronic Sciatica. Rheumatology 
1970;10(7):368-374.

12. MACNAB I. Negative Disc Exploration. The Journal of Bone \& Joint Surgery 1971;53(5):891-903.

13. Complications of fluoroscopically-guided spinal injections. Archives of Physical Medicine and Rehabilitation 1995;76(11):1032.

14. Weiner B, Fraser R. Foramina Injection for Lateral Lumbar Disc Herniation. The Journal of Bone and Joint Surgery British 1997;79-B(5):804-807.

15. Weber H. Volvo Award in Clinical Sciences. Lumbar disc herniation. A controlled, prospective study with ten years of observation. Spine 1982;8:131-40.

16. Narozny Martin, et al. Therapeutic Efficacy of Selective Nerve Root Blocks in the Treatment of Lumbar Radicular Leg Pain. Swiss Med Wkly 2001;131(5-6):75-80.

17. Pfirrmann CW, Oberholzer PA, Zanetti M, et al. Selective nerve root blocks for the treatment of sciatica: evaluation of injection site and effectiveness--a study with patients and cadavers. Radiology 2001;221(3):704711. doi:10.1148/radiol.2213001635

18. Cuckler JM, Bernini PA, Wiesel SW, Booth RE Jr, Rothman RH, Pickens GT. The use of epidural steroids in the treatment of lumbar radicular pain. A prospective, randomized, double-blind study. J Bone Joint Surg Am. 1985;67(1):63-66.

19. Ernst Edzard. Acupuncture for Chronic Low Back Pain. The New England journal of medicine. 363. 1776; author reply 2010, 1777-8.

20. El-Khoury GY, Ehara S, Weinstein JN, Montgomery WJ, Kathol MH. Epidural steroid injection: A procedure ideally performed with fluoroscopic control. Radiology 1988;168(2):554-557. https://doi.org/10.1148/radiology.168.2.2969118

21. Bogduk N, Christophidis N, Cherry D. Epidural use of steroids in the management of back pain. Report of working party on epidural use of steroids in the management of back pain. National Health and Medical Research Council. Canberra, Commonwealth of Australia, 1994, 1-76.

22. Pandey RA. Efficacy of Epidural Steroid Injection in Management of Lumbar Prolapsed Intervertebral Disc: A Comparison of Caudal, Transforaminal and Interlaminar Routes. J Clin Diagn Res 2016;10(7):RC05-RC11. doi:10.7860/JCDR/2016/18208.8127.

23. Salunkhe R, Pisal T, Hira Y, Singh A, Patel J, Goud S. Study of nerve root block procedure as a diagnostic and therapeutic aid in lumbosacral radiculopathy. Indian $\mathbf{J}$ Pain 2016;30:127. 